\section{EU-Recht will Diabetiker drangsalieren Vorm Führerschein zum Zucker-TÜV?}

> Vor der Führerscheinbeantragung zum Gutachter? Das könnte Ihren Diabetikern in Zukunft blühen, nämlich dann, wenn voraussichtlich 2013 die EUFührerscheinrichtlinien in nationales Recht umgesetzt werden. Dabei sollte der ausstellende Gutachter nicht der behandelnde Arzt sein. Berücksichtigt wird dabei Blutzuckertagebuch, $\mathrm{HbA}_{1 c}$-Wert sowie eine körperliche Untersuchung.

Zur Zeit gehört der Diabetes nicht zu den meldepflichtigen Krankheiten. Die

\section{DDG: Nicht unkritisch ans Magenband!}

> Die Deutsche Diabetes-Gesellschaft (DDG) warnt: Magenbypass-Chirurgie zur Typ-2-Diabetes-Therapie ist langfristig nicht ausreichend evaluiert. Nach Angaben der Expertengruppe „Metabolische Chirurgie“ werden durch MagenOps. 70\% der extrem Übergewichtigen von Stoffwechselschäden befreit, weshalb die Befürworter die Methode auch schon bei einem BMI unter $30 \mathrm{~kg} / \mathrm{m}^{2}$ anwenden wollen. Dieser Forderung widerspricht die DDG, da hierfür bislang noch keine wissenschaftlich hochwertigen Studien vorliegen. Derzeit sei noch nicht abzusehen, ob operierte Diabetiker langfristig besser, komplikationsärmer und länger leben. Pressemitteilung DDG

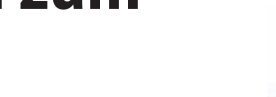

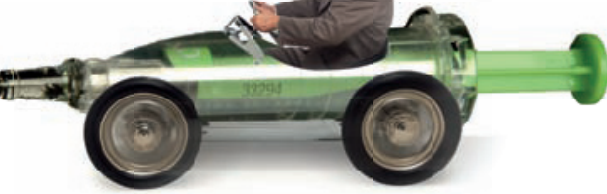

Mit Diabetes einfach so durchstarten? Das könnte in Zukunft schwierig werden.

Behörde kann allerdings eine entsprechende Bescheinigung fordern z.B. nach zu schnellem Fahren oder Unfall wegen einer Unterzuckerung.

(sko)

Pressemitteilung DDG

\section{Diabetes-Epidemie rollt wie Tsunami}

> „Die globale Diabetes-Epidemie entwickelt sich wie ein Tsunami“, erklärt der Präsident der Internationalen DiabetesFöderation (IDF) Jean Claude Mbanya aus Kamerun. Weltweit leben 2011 bereits 366 Mio. Diabetiker, pro Jahr sterben 4,6 Mio. Menschen an Diabetes-Folgen. Die globalen jährlichen Kosten belaufen sich auf 465 Milliarden Dollar, so aktuelle Zahlen aus der fünften Neuauflage des globalen Diabetes-Atlas. Die IDF möchte mit einem globalen Action-Plan gegensteuern, der Aufklärung, Prävention und den Aufbau von Versorgungsstrukturen für Diabetiker in Ländern mit niedrigen Einkommen in den Mittelpunkt stellt. EASD-Tagung Lissabon, September 2011

\title{
Backen für die Wissenschaft
}

Es gibt schon lustige Studien: In Toronto z.B. wurde 117 Typ-2-Diabetikern zur Erprobung einer hilfreichen Ernährung entweder eine 75-Gramm-Mischung aus Mandeln, Pistazien, Wallnüssen, Pekannüssen, Cashews und Macadamianüssen gereicht, das zweite Drittel durfte statt dessen Muffins essen, das letzte Drittel wurde mit je halbe halbe verköstigt. Und tatsächlich: Die Nussgruppe schnitt sowohl bezüglich $\mathrm{HbA}_{1 c}$-Senkung als auch Blutfettwerte signifikant besser ab - bei Muffins als Vergleichskost vielleicht auch kein Wunder. Wer sich da doch lieber auf die bewährte Medizin verlassen will, findet in unserem Schwerpunkt eine aktuelle Übersicht, welches Antidiabetikum für welchen Patienten am besten geeignet ist, wie es um die Insulintherapie bei herzkranken Diabetikern bestellt ist und was vom Screening bei Gestationsdiabetes zu halten ist. Und wenn Sie dann schlauer sind, haben Sie sich auch einen kleinen Muffin zur Belohnung verdient. Eine angenehme Lektüre wünscht
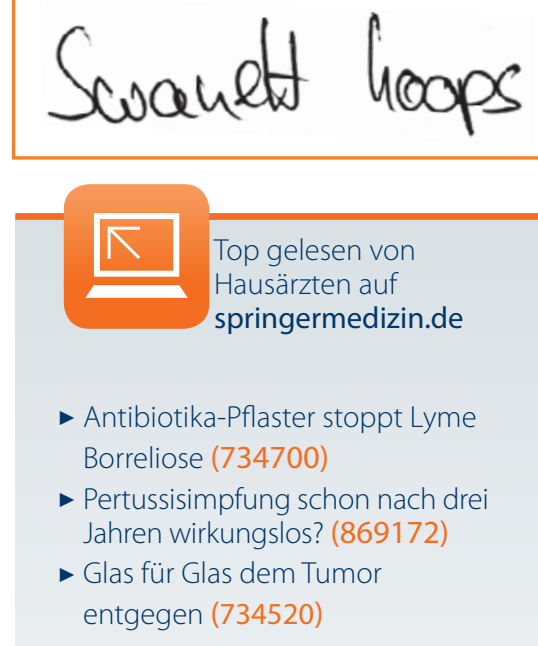

Loggen Sie sich ein und nutzen Sie die Fülle von Beiträgen auf springermedizin.de! Die oben genannten Artikel finden Sie, in dem Sie die ID-Nr. in die Suche eingeben. 